\title{
Représentations linguistiques d'élèves du secondaire à l'égard de la langue dans les contextes familier, scolaire et en sciences
}

\author{
Stéphanie Melanson \\ Marianne Cormier \\ Université de Moncton
}

\begin{abstract}
Résumé
Les élèves vivant en milieu francophone minoritaire rencontrent quotidiennement différentes langues et différents registres de langue. Alors que leur contexte de vie familial et social fait usage d'un registre plus familier, l'école favorise un registre plus soutenu et spécialisé, particulièrement dans les cours de sciences. Cette étude s'intéresse à décrire et à comprendre les représentations linguistiques que se font les élèves dans ces différents contextes. Quatorze élèves d'une classe de sciences de 10e année ont été interrogés au sujet de leurs représentations linguistiques et ont été observés lors de la pratique d'activités langagières en salle de classe. Les résultats indiquent que les élèves ont différentes représentations des langues et registres qu'ils utilisent quotidiennement dans leurs divers contextes de vie. Par ailleurs, les élèves ne semblent pas conscients du rôle des activités langagières dans leur apprentissage en sciences. Les répercussions de ces résultats sur la pédagogie sont brièvement discutées.
\end{abstract}

\section{Abstract}

Francophone minority students come in contact with different languages and language registers on a daily basis. While the register used in familial and social contexts is rather familiar, the language used at school is relatively formal and specialized, particularly in science teaching. This study seeks to describe and understand students' linguistic representations regarding those different contexts. Fourteen grade 10 students were interviewed pertaining to their linguistic representations and were also observed during activities in science class that integrated language and science content. Results show that students hold different representations of the languages they use daily. Furthermore, students did not seem aware of the benefits that language activities may bring to their science learning. The pedagogical impact of these results is briefly discussed. 


\section{Introduction}

En sciences, les élèves francophones du Nouveau-Brunswick ont obtenu de faibles résultats lors des dernières évaluations du programme international pour le suivi des acquis (PISA) et du programme d'indicateurs du rendement scolaire (PIRS). Ces résultats étaient significativement inférieurs à la moyenne nationale canadienne des élèves francophones et anglophones, et à ceux des élèves anglophones du Nouveau-Brunswick (Buissière, Knighton et Pennock, 2007; Conseil des ministres de l'Éducation du Canada [CMEC], 2004). Puisque les résultats de ces élèves s'avèrent également faibles en lecture, certains auteurs sont d'avis que ces difficultés en sciences pourraient être attribuées aux limites langagières chez ces élèves (CMEC, 2004; Cormier, Pruneau et Rivard, 2004; Pruneau et Langis, 2002).

En raison de leur milieu de vie anglodominant, les élèves francophones du Nouveau-Brunswick ont moins d'occasions de socialisation dans la langue minoritaire et ont souvent peu d'expériences littératiées avec celle-ci (Hamers et Blanc, 2000; Landry, 2003b). L'attrait de l'anglais est grandissant et les médias anglo-américains sont de plus en plus présents dans les foyers. Dans ces communautés, le paysage linguistique est fortement anglophone (Cormier, 2005; Landry, 2003b; Landry et Allard, 1999). L'exogamie, c'est-à-dire le mariage de deux individus de langue et de culture différentes, est un phénomène de plus en plus présent dans les foyers de ces milieux et peut aussi influencer les compétences langagières des élèves. Selon Landry (2003a), $64 \%$ des enfants d'ayants droit ${ }^{1}$ francophones au Canada vivent dans une famille exogame. Chez l'ensemble des enfants qui vivent dans des familles exogames, seulement $33 \%$ utilisent le français au moins régulièrement à la maison et seulement $45 \%$ déclarent avoir une connaissance du français.

Peu importe le milieu dans lequel ils vivent, les élèves rencontrent quotidiennement divers registres de langues selon la situation dans laquelle ils se retrouvent (Yore, 2008). Legendre (2005) précise que les locuteurs font usage de différentes formes linguistiques dépendamment des interlocuteurs, du contexte et de l'intention de communication. Ainsi, dans leur entourage familier, à la maison et avec leurs amis, les élèves font usage de leur registre vernaculaire, un registre familier, avec un accent et des expressions typiques de la région. L'école, toutefois,

\footnotetext{
${ }^{1}$ Le terme ayants droit désigne généralement les parents qui répondent aux critères de l'article 23 de la Charte canadienne des droits et libertés. Conséquemment, ce sont les parents qui ont droit à l'école, pour leurs enfants, de la langue officielle minoritaire de leur province, et à la gestion de celle-ci (Rocque, 2008).
} 
met à profit un registre plus soutenu, propice à des communications formelles, ce registre étant à la fois la langue véhiculaire des contenus d'enseignement, et la langue cible que l'on souhaite faire acquérir aux élèves. De plus, chaque matière scolaire a son registre spécialisé, avec des expressions propres au domaine d'études et des formulations spécifiques. Particulièrement en sciences, un vocabulaire spécialisé est d'usage, et ce registre spécialisé scientifique s'entrecoupe de diagrammes, d'expressions mathématiques, de schémas et dessins. Parfois, certains mots changent de sens d'un registre à l'autre, comme le mot "énergie » qui prend un sens assez large dans le registre vernaculaire, mais qui a un sens très précis dans l'étude de la physique. D'autre fois, une même réalité s'exprime différemment d'un contexte à un autre : migraine, mal de tête, mal aux cheveux. La navigation entre registres, c'est-à-dire le passage plus ou moins spontané d'un locuteur d'un registre de langue à l'autre en fonction du contexte, peut donc s'avérer un défi, particulièrement en milieu minoritaire, lorsque le registre vernaculaire s'éloigne beaucoup du registre soutenu favorisé par l'école et que, par ailleurs, les élèves naviguent également entre deux langues, dans ce cas, le français et l'anglais (Rivard et Cormier, 2008).

Afin de s'approprier le registre spécialisé partagé par un groupe social (les scientifiques, par exemple), un individu doit avoir l'occasion de baigner dans les pratiques sociales et langagières de ce groupe et de s'immerger dans les contextes d'utilisation propres à ce registre. Une représentation positive du registre et une reconnaissance de sa valeur facilitent son appropriation (Gee, 2002). II apparaît donc important de connaître le caractère positif ou négatif des représentations linguistiques des élèves du sud-est du Nouveau-Brunswick quant à leurs différents registres de langue. Des individus porteurs de représentations linguistiques négatives pourraient entretenir une résistance à l'appropriation de nouveaux registres spécialisés utilisés à l'école et dans les cours de sciences. Une compréhension de la valeur des représentations associées aux différents registres vient mieux renseigner les enjeux pédagogiques en milieu linguistique minoritaire. Puisque les représentations linguistiques peuvent aider à comprendre le comportement des locuteurs, une meilleure compréhension de ces représentations pourrait expliquer le succès ou le non-succès d'une activité pédagogique qui intègre la langue et les sciences. Ainsi, une activité littératiée en sciences pourrait ne pas être pleinement exploitée si les élèves détiennent des représentations négatives à l'égard de leurs registres de langue.

Dans cet article, dans un premier temps, nous présentons les différents concepts théoriques encadrant cette recherche, dont ceux de représentations linguistiques, d'insécurité linguistique, de registre de langue spécialisé utilisé en sciences et d'usage de la langue en sciences. Deuxièmement, nous présentons les résultats d'une étude de cas réalisée dans le cadre d'une recherche exploratoire effectuée avec des élèves d'une classe de 10e année du sud-est du Nouveau-Brunswick, afin de décrire et comprendre les représentations qu'ils se font de leurs différents registres linguistiques. Nous discutons finalement, en conclusion, des enjeux pédagogiques qui découlent d'une compréhension élargie des représentations linguistiques.

\section{Les représentations linguistiques}

Alors que la sociolinguistique étudie les rapports entre la langue (ou les langues), ses locuteurs et la société, un courant particulier de cette science étudie les phénomènes linguistiques en tant que pratiques sociales (Boudreau, Dubois et d'Entremont, 2008). Un domaine important de ce courant s'intéresse aux représentations linguistiques 
(Blanchet et Robillard, 2003; Calvet, 1999; Klinkenberg, 2001) qui se composent de croyances relativement stables que les locuteurs se construisent au sujet de leurs pratiques linguistiques à la suite de leurs expériences avec la langue dans divers contextes sociaux. Ces croyances déterminent les évaluations que les locuteurs font de leur propre langue, de même que leurs attitudes envers les langues. Les représentations linguistiques se construisent essentiellement dans le discours (Boudreau, Dubois et d'Entremont, 2008; Calvet, 1999). Incluant l'ambiance linguistique et culturelle dans le milieu familial, scolaire et social et les perceptions des individus concernant le statut de leur langue, le vécu sociolinguistique des individus influence leurs représentations linguistiques (Boudreau et Perrot, 2005; Gee, 2008). Les représentations linguistiques se façonnent selon ce que les locuteurs pensent de leurs pratiques linguistiques, comment ils évaluent leurs pratiques linguistiques par rapport à celles des autres et comment ils évaluent leur langue en rapport avec les autres langues (Calvet, 1999). En s'inspirant des travaux de Bourdieu (1982) et de Labov (1972), Francart (1997) explique que, souvent, à la suite des comparaisons qu'ils font entre euxmêmes et d'autres catégories de locuteurs, les individus peuvent se construire des représentations linguistiques négatives, car ils perçoivent un écart entre leur parler et celui d'un autre groupe qu'ils se représentent comme étant plus légitime. Le constat de disparités et la construction de représentations linguistiques négatives peuvent générer des insécurités linguistiques. Boudreau, Dubois et d'Entremont (2008) affirment que le positionnement d'un individu entre les pôles de sécurité et d'insécurité linguistiques devient une donnée incontournable pour aider à comprendre les comportements et les pratiques linguistiques. Pour approfondir la compréhension des représentations linguistiques des individus, il est donc important de considérer comment ceux-ci décrivent et évaluent leurs propres pratiques linguistiques, comment ils les comparent aux pratiques linguistiques des autres, de même que de cerner leurs perceptions et leurs croyances au sujets des différents registres de langue auxquels ils sont exposés.

Au sud-est du Nouveau-Brunswick, le chiac est utilisé par la majorité des gens (Boudreau et Perrot, 2005). II s'agit d'une variété interlectale «qui se caractérise avant tout par l'intégration, au sein d'une matrice acadienne, d'abondants emprunts lexicaux, morphologiques, et syntaxiques à l'anglais » (Boudreau et Perrot, 2005, p. 9). Pour les besoins de cet article, nous parlerons donc du chiac comme d'une variété de langue utilisée principalement dans le registre vernaculaire. Les locuteurs du chiac entretiennent différentes représentations à son égard. Certaines recherches ont été effectuées dans la région afin de mieux comprendre comment les gens se représentent cette variété vernaculaire. Ainsi, parler chiac peut être considéré comme l'expression d'une affirmation identitaire forte, ou comme une incapacité de bien s'exprimer (Boudeau, 1996, Boudreau et Perrot, 2005; Boudreau, Dubois et d'Entremont, 2008). Dans une recherche effectuée par Boudreau (1996), les jeunes de Moncton, au NouveauBrunswick, affirment que leur manière de parler n'est pas optimale. En fait, certains jeunes sont d'avis que l'usage du chiac n'est pas approprié pour certaines situations plus formelles. Ils sentent toutefois le besoin de l'utiliser avec leurs amis parce qu'ils veulent «faire comme tout le monde ». Une autre recherche effectuée par Perrot (2006) obtient des résultats semblables. Les jeunes de Moncton dénigrent l'utilisation de leur variété linguistique régionale en affirmant que le chiac n'est pas une bonne manière de parler. Toutefois, certains jeunes peuvent faire ressortir des éléments positifs du chiac liés à l'héritage et à la culture acadienne. 


\section{L’insécurité linguistique}

Les élèves qui abaissent leur façon de s'exprimer ou qui ne se sentent pas capables de bien parler pourraient vivre une insécurité linguistique. Boudreau, Dubois et d'Entremont (2008) définissent deux types d'insécurité linguistique : statutaire et formelle. L'insécurité linguistique statutaire est liée au sentiment que sa langue est moins prestigieuse qu'une autre langue. Par exemple, un locuteur étant d'avis que le français est une langue moins « importante » que l'anglais pourrait vivre une insécurité statutaire. L'insécurité formelle est liée au sentiment de ne pas être capable de bien parler sa langue. Un locuteur parlant français, mais pensant qu'il n'utilise pas les mots appropriés ou qu'il est tout simplement « mauvais » dans cette langue pourrait vivre une insécurité formelle.

Si elle est présente chez les élèves en salle de classe, comme le suggèrent les recherches de Boudreau (1996) et Perrot (2006) dans le cas des élèves du Nouveau-Brunswick, l'insécurité linguistique peut influencer le choix de s'exprimer ou non devant un groupe ou même de participer ou non à toute activité langagière. Daley (2003) soulève que, paradoxalement, de par sa mission de reproduire et de maintenir la langue française, l'école du milieu linguistique minoritaire mise surtout sur un registre plus soutenu. Puisque la langue qu'elle valorise s'éloigne du vernaculaire de la majorité de ses élèves, l'école ne vise pas réellement la reproduction de leur langue, mais plutôt sa normalisation et sa standardisation. Daley (2003) fait donc remarquer qu'un paradoxe langagier s'installe alors que la langue qui symbolise l'identité de ses locuteurs est exclue de l'école. Or, comme le souligne cette auteure, la perception du chiac évolue et est de moins en moins considérée comme une erreur à corriger, mais plutôt comme une base linguistique sur laquelle construire. De même, Boudreau, Dubois et d'Entremont (2008) proposent de construire sur les acquis linguistiques vernaculaires. Cormier (2005) abonde dans ce sens et recommande, afin d'amener les élèves à contrer l'insécurité linguistique, que les enseignantes et les enseignants s'assurent d'adopter des pratiques pédagogiques propices au développement d'un rapport positif avec la langue. Les élèves doivent avoir la chance de vivre de nombreuses expériences positives avec la langue. Les enseignantes et les enseignants doivent encourager les élèves à s'exprimer et doivent avoir une attitude ouverte à l'égard du registre vernaculaire utilisé par les élèves. Pour faciliter ce travail, il semble important tout d'abord d'approfondir les connaissances sur les représentations linguistiques qu'entretiennent les élèves.

\section{Registre spécialisé et usage de la langue en sciences}

Les considérations qui précèdent montrent la légitimité de se demander comment les représentations linguistiques et l'insécurité linguistique viennent influencer l'apprentissage en sciences. En réponse à cette question, il convient de décrire la spécificité du registre de langue spécialisé utilisé en sciences, pour ensuite discuter de la pertinence et de l'importance des activités langagières en sciences.

En sciences, on utilise un registre spécialisé composé de mots, d'images, de graphiques, de diagrammes, de figures et de symboles qui sont spécifiques au domaine (Wellington et Osborne, 2001). D'après Rivard et Cormier (2008), les scientifiques font partie d'une communauté discursive distincte qui emploie un registre de langue spécifique à leur groupe social. Les élèves doivent ainsi avoir la chance de s'approprier le registre spécialisé des sciences afin de pouvoir accéder aux savoirs propres aux divers domaines des sciences. 
En milieu minoritaire francophone, puisque les élèves sont fréquemment exposés à l'anglais à l'extérieur de l'école, ces derniers ont moins de possibilités de s'approprier le registre de langue soutenu favorisé par l'école et le registre spécialisé des sciences. Pour combler ce manque, il est fondamental pour les enseignants d'inclure des activités littératiées en sciences afin de permettre aux élèves d'être exposés au registre spécialisé des sciences et de pouvoir l'acquérir (Laplante, 2001, Rivard et Cormier, 2008). En effet, les auteurs s'entendent sur le fait qu'il faut être exposé au registre spécialisé des sciences afin de se l'approprier (Cormier, Pruneau et Rivard, 2004, Laplante, 2001, 2007; Pruneau et Langis, 2003). Les élèves doivent avoir la possibilité de "parler » comme des scientifiques en participant à des activités langagières en sciences. Les habiletés de lecture et d'expression orale et écrite sont essentielles à l'apprentissage en sciences (Gibbons, 1998; Laplante, 2001; Rivard et Straw, 2000; Yore, Hand et Prain, 2002). La langue est une partie intégrale de cet apprentissage : les scientifiques discutent leurs hypothèses, prennent note de leurs observations et communiquent, oralement et par écrit, les résultats de leurs travaux (Norris et Philips, 2003). C'est donc en permettant aux élèves de vivre fréquemment des activités d'écriture, de lecture et de discussion que ces derniers développent les habiletés langagières nécessaires à l'apprentissage en sciences.

Les activités littératiées sont toutefois peu présentes dans les classes de sciences (Osborne, 2002), peu importe qu'il s'agisse d'activités d'écriture, de discussion ou de lecture. Même si l'écriture est souvent utilisée dans les salles de classe, les élèves demeurent la plupart du temps très passifs lorsqu'ils effectuent les tâches d'écriture communes (Wellington et Osborne, 2001). Par exemple, l'élève qui copie simplement des notes inscrites au tableau ne fait pas appel à des processus cognitifs très complexes. L'écriture peut être une activité bénéfique si elle fait appel à la clarification d'idées, à la construction et à la structuration des savoirs ou encore, à la métacognition (Cormier, 2004; Hand et Prain, 2006; Rivard, 1994).

La discussion, quant à elle, est une activité bénéfique, surtout lorsqu'elle est accompagnée d'une activité d'écriture (Rivard et Straw, 2000). Cependant, pour être efficace, la discussion doit engendrer la réflexion profonde de la part des élèves et ne doit pas être simplement menée par l'enseignante ou l'enseignant qui pose des questions et attend la réponse des élèves. Le partage d'idées doit être riche afin que les élèves aient la possibilité de s'approprier le vocabulaire propre au registre spécialisé des sciences (Wellington et Osborne, 2001).

Tout comme les activités d'écriture, les activités de lecture sont encore souvent très passives et réalisées de manière individuelle. Par exemple, l'élève qui lit une section de son manuel scolaire afin de compléter un devoir ne bénéficie pas des bienfaits de la lecture en sciences. Or, la lecture peut s'avérer une activité pouvant amener les élèves à faire appel à leurs connaissances et expériences antérieures et à des stratégies cognitives et métacognitives (Wellington et Osborne, 2001). Les élèves doivent être capables de faire des inférences et d'interpréter de différentes manières les textes scientifiques tout en reconstruisant le sens de ceux-ci (Norris et Phillips, 2003).

\section{Objectif et questions de recherche}

La faiblesse des compétences langagières des élèves de milieux minoritaires francophones du NouveauBrunswick pourrait être un facteur contribuant aux faibles résultats obtenus par ces élèves en sciences lors des derniers tests PIRS et PISA. Le vécu sociolangagier des élèves et surtout leurs représentations linguistiques pourraient avoir un impact sur le déroulement des activités langagières en salle de classe et affecter la capacité des 
élèves d'en tirer profit. De nombreuses recherches (Gibbons, 1998; Hand et Prain, 2006; Laplante, 2001; Lee et Fradd, 1996; Rivard et Straw, 2000; Yore, Hand et Prain, 2002) ont déjà montré l'importance des compétences langagières dans l'apprentissage et l'enseignement des sciences. Toutefois, l'incidence des représentations linguistiques sur les pratiques et les comportements linguistiques des élèves (Boudreau, 1996) et, par ricochet, sur le succès des activités langagières qui leur sont proposées en sciences demande à être explorée davantage. La présente étude vise alors à décrire et comprendre les représentations linguistiques d'élèves du secondaire à l'égard de leur registre de langue vernaculaire utilisé à la maison, du registre soutenu utilisé à l'école et du registre spécialisé utilisé en sciences, ces trois registres étant intimement interreliés. Ensuite, en tenant compte de ces représentations linguistiques, il convient de décrire comment les élèves vivent des activités intégrant la langue en sciences. Cette recherche pourrait ainsi contribuer à comprendre davantage les enjeux de l'enseignement et de l'apprentissage des sciences en milieu minoritaire francophone. Plus spécifiquement, la présente recherche veut répondre aux questions suivantes :

1) Comment des élèves d'une classe de $10^{e}$ année du secondaire de la région de Moncton se représententils les différents registres langagiers: familier ou vernaculaire, soutenu de l'école et spécialisé des sciences?

2) À la lumière de ces représentations linguistiques, comment ces mêmes élèves vivent-ils les activités langagières en sciences? Est-ce que ces élèves utilisent la langue comme un outil d'apprentissage ? Comment?

\section{Méthodologie}

Étant donné la visée compréhensive de cette étude exploratoire, nous avons adopté une méthodologie qualitative. «La recherche qualitative en sciences humaines a comme but premier de comprendre les phénomènes sociaux, soit des groupes d'individus ou des situations sociales » (Poisson, 1991, p. 12). II s'agit de saisir l'essentiel de la réalité sociale étudiée en interprétant les caractéristiques du milieu sans toutefois s'imposer de limites. La recherche qualitative s'intéresse surtout à des échantillons et à des cas plus restreints, mais les étudie plus en profondeur (Deslauriers, 1991). Ainsi, pour comprendre comment les élèves se représentent leurs divers registres langagiers et comment ils utilisent la langue dans les cours de sciences en tant qu'outil d'apprentissage, nous avons fait une étude de cas des élèves d'une classe de $10^{\circ}$ année. L'étude de cas est une méthodologie de recherche de plus en plus utilisée en éducation (Karsenti et Savoie-Zajc, 2004).

Nous décrivons donc ici les participants à cette recherche, le déroulement de l'étude de cas, les outils de collecte de données et les méthodes d'analyse des données.

\section{Participants}

Cette recherche a été réalisée dans la seule école secondaire de la ville de Moncton, au sud-est du Nouveau-Brunswick, une région majoritairement anglophone avec un taux de croissance démographique très élevé. Répondant à une invitation lancée au personnel de l'école spécialisé dans l'enseignement des sciences, un enseignant d'une classe de 10e année a accepté de participer à l'étude. Parmi les 26 élèves de la classe, 14 (6 filles et 8 garçons) ont accepté volontairement de participer à la recherche, en accord avec les procédures éthiques en vigueur au Canada. Le profil sociodémographique de ces élèves a été obtenu par voie de questionnaire. Ainsi, parmi les 
participantes et participants, 8 sont issus de familles exogames, alors que les 6 autres sont de familles endogames (dont 2, de familles immigrantes). Les participantes et participants ont donc tous un vécu sociolangagier unique. Trois déclarent l'anglais comme langue maternelle alors que pour les autres, la langue maternelle est le français. La plupart sont nés et ont grandi dans la région de Moncton, mais quelques-uns sont nés ailleurs et sont arrivés à Moncton à différents moments de leur vie.

\section{Le déroulement de l'étude de cas}

L'étude de cas s'est déroulée en deux étapes: 1) l'observation lors d'activités différentiées intégrant la langue en sciences et 2) la tenue d'entrevues avec les élèves participants.

Les élèves ont été observés lors d'activités différentiées intégrant la langue en sciences durant 4 leçons de 90 minutes. Au cours de ces activités, les élèves travaillaient en sous-groupes et devaient accomplir différentes tâches intégrant la lecture, l'écriture et la discussion. Typiquement, les élèves devaient lire dans leur manuel et discuter entre eux afin de résoudre un problème commun. Ils devaient, à l'occasion, effectuer des manipulations avec du matériel afin de découvrir certains phénomènes chimiques. Chaque sous-groupe visait les mêmes objectifs, même si les tâches étaient différentes d'un sous-groupe à l'autre. Lors de ces activités, l'observatrice a effectué une observation libre en se déplaçant constamment d'un sous-groupe à l'autre. Elle a évité d'interagir avec les élèves, sauf lorsque ces derniers lui ont posé des questions directement. À la fin de chacune des leçons, tous les élèves devaient écrire dans leur journal réflexif et, après avoir complété les 4 leçons, ils ont subi une évaluation formative.

Après avoir complété les observations des 4 leçons, chaque élève participant a été invité à prendre part à une entrevue individuelle semi-dirigée. Ces entrevues se sont déroulées durant le temps de classe, dans un local de l'école. Elles ont duré en moyenne 15 minutes et elles ont été enregistrées.

\section{Les outils de collecte de données}

Comme le laisse entendre la description du déroulement de l'étude de cas, plusieurs outils de collecte de données ont servi lors de cette recherche : les profils sociodémographiques des élèves, le journal de bord de la chercheure, l'entrevue semi-dirigée, les journaux réflexifs des élèves ainsi que les évaluations formatives.

\section{Les profils sociodémographiques}

Les 14 élèves participants ont rempli un profil sociodémographique permettant de recueillir des informations au sujet de leur vécu sociolangagier. Notamment, "À la maison, ma mère parle... », "Avec mes amis, je parle... » sont des exemples de questions qui figurent dans le questionnaire du profil pour les élèves. Ce questionnaire a été administré avant d'effectuer les observations en salle de classe.

\section{Le journal de bord de la chercheure}

Au courant des 4 leçons observées, la chercheure principale a prêté attention aux comportements langagiers des élèves lorsqu'ils ont participé aux activités langagières en classe de sciences et les a notés dans son journal de bord. Elle a pu ainsi noter le discours des élèves et leurs comportements lors des activités en se concentrant, entre autres, sur l'aisance avec laquelle les élèves s'exprimaient dans leur groupe, l'utilisation des outils langagiers mis à la 
disposition des élèves, l'aide nécessitée lors des tâches impliquant la langue et l'usage du registre spécialisé des sciences. La chercheure a également noté ses observations sur le comportement langagier des élèves lors des entrevues.

\section{Les entrevues semi-dirigées}

L'entrevue individuelle effectuée avec chaque participant visait à obtenir des informations au sujet de son vécu sociolangagier, son autoévaluation de ses compétences langagières à la maison, à l'école et en sciences, ainsi que ses croyances à l'égard des différents registres langagiers. Les questions d'entrevues étaient donc regroupées en 4 thèmes. Le premier thème portait sur le registre familier des élèves (Comment me décrirais-tu la manière dont tu parles à la maison? Comment te sens-tu par rapport à la manière dont tu parles avec tes amis?), le deuxième, sur le registre soutenu favorisé par l'école (Comment me décrirais-tu la manière dont tu parles à l'école?) et le troisième, sur le registre spécialisé des sciences (Décris-moi le langage utilisé en sciences. Comment te sens-tu lorsque tu dois écrire, lire ou discuter en sciences?). Finalement, le quatrième thème portait plus précisément sur les activités langagières vécues dans les cours de sciences durant les semaines précédant l'entrevue (Comment as-tu vécu ces activités? Quels ont été les aspects positifs/négatifs de ces activités?).

\section{Les journaux réflexifs}

Lors des 4 leçons intégrant les activités langagières en sciences, les élèves ont noté leurs réflexions et les apprentissages effectués dans un journal réflexif. Un journal réflexif peut être un outil d'apprentissage très efficace. De plus, le journal peut favoriser le développement des habiletés métacognitives en permettant aux élèves de réfléchir au sujet de leurs découvertes, de formuler des hypothèses et de critiquer leurs idées (Hammond, 2002).

Afin d'aider les élèves à rédiger leur journal réflexif, l'enseignant leur a proposé les questions suivantes : «Qu'as-tu appris/découvert aujourd'hui ?» et « Qu'as-tu trouvé difficile/facile lors de l'activité ? ». L'écriture dans le journal réflexif a servi de complément aux discussions qui ont eu lieu lors de chaque activité langagière et a été utilisée à la fois comme outil pédagogique et comme outil de collecte de données.

\section{Les évaluations formatives}

Après avoir complété les 4 leçons intégrant les activités langagières, l'enseignant a administré un test aux fins d'évaluation formative. Les élèves devaient répondre à des questions au sujet des acides et des bases. Ensuite, ils se sont autocorrigés et ont ainsi pu se rendre compte des éléments bien maîtrisés et de ceux qui étaient moins bien maîtrisés. Ces évaluations formatives ont été recueillies et utilisées comme données non pas pour vérifier l'efficacité des activités langagières sur l'apprentissage des élèves, mais pour relever les particularités de leur expression écrite.

\section{Analyse des données}

Afin de répondre aux questions de recherche, nous avons eu recours à deux techniques différentes d'analyse, 1) une analyse de contenu des entrevues semi-dirigées, qui visait à répondre à la première question de recherche et 2) la construction et l'analyse de récits narratifs, ciblant la deuxième question de recherche, et mettant à profit l'ensemble des données recueillies. 
L'analyse du contenu a débuté par une lecture préliminaire des transcriptions du verbatim des entrevues pour en faire ressortir les éléments clés. Ensuite, la codification des données (Mayan, 2001), à l'aide du logiciel Atlas.ti, a été réalisée en découpant chaque transcription en unité de sens. Afin d'éviter d'interpréter trop rapidement les propos des élèves, les codes étaient simplement des reformulations de leurs propos. Par exemple, le code «Emmanuel: Son père utilise toujours un bon vocabulaire » peut être associé à une partie du verbatim tel que: «...mon père utilise toujours des bons vocabulaires...». Ensuite, afin de rendre les données plus signifiantes, ces codes ont été regroupés en cinq grandes catégories : comportements langagiers à la maison et avec les amis; comportements langagiers à l'école; comportements langagiers en sciences; autres comportements langagiers; vécu des élèves lors des activités langagières.

D’après Mayan (2001), les catégories doivent inclure toutes les données et bien représenter celles-ci. L'analyse du contenu est un processus circulaire qui amène le chercheur à réévaluer ses choix, à ajouter d'autres catégories ou à créer d'autres liens entre les données. Ainsi, à la suite de la première catégorisation, et étant donné que les catégories étaient très vastes et regroupaient un trop grand nombre de codes, il s'est avéré pertinent de procéder à une sous-catégorisation des données. Chaque catégorie a été subdivisée en fonction des souscatégories suivantes : situation langagière dans ce contexte; description de l'élève de cette langue; autoévaluation de cette langue; perception de cette langue; justification de cette perception.

La dernière étape de l'analyse du contenu a consisté à établir des liens entre les catégories, à faire ressortir les traits communs et à tirer des conclusions. Les informations ont été regroupées dans des tableaux et les propos des élèves concernant leurs comportements langagiers dans les différents contextes ont été comparés afin de cerner leurs représentations à l'égard de leur langue à la maison, à l'école et en sciences.

La construction de récits narratifs a ciblé 3 élèves participants à cette étude et visait à répondre à la deuxième question de recherche. Selon Karsenti et Savoie-Zajc (2004), les textes narratifs viennent compléter la phase de l'analyse des données lors d'une étude de cas. Ce genre de récit permet de mettre à profit, de réduire et de résumer les données recueillies à partir de différentes sources. Les trois élèves ont été choisis en raison des différences marquées dans leur vécu sociolangagier et dans leur rendement scolaire. Chaque récit a permis de mieux illustrer les représentations linguistiques des élèves, leurs comportements langagiers, leurs réactions et leurs commentaires lors des activités intégrant la langue. L'analyse comparative de ces 3 textes narratifs a ensuite servi à mieux comprendre comment les représentations des élèves à l'égard des différents registres de langue peuvent avoir un impact sur leurs comportements lors d'activités intégrant la langue en sciences.

\section{Résultats}

Les résultats se présentent en deux grandes parties : premièrement, il s'agit de décrire les différentes représentations qu'ont les élèves à l'égard des registres de langue qu'ils utilisent et comment ils composent avec ceuxci. Deuxièmement, l'analyse comparative des textes narratifs décrivant le vécu de 3 élèves lors des activités intégrant la langue en sciences permet de voir s'ils utilisent la langue comme un outil d'apprentissage en sciences. 


\section{Représentations linguistiques}

Les élèves évoquent deux langues et une variété de langue, notamment le français, le chiac et l'anglais, qu'ils utilisent quotidiennement dans les contextes familial/social, scolaire et en sciences.

\section{Représentations linguistiques du français}

Le français est décrit de plusieurs façons et les élèves semblent en avoir différentes représentations. Certains élèves perçoivent le français comme une langue difficile ayant beaucoup de règles de grammaire, mais qui est nécessaire pour se débrouiller dans la vie de tous les jours. Dans le contexte familial/social, contexte associé au registre vernaculaire, 11 élèves affirment utiliser le français (les 3 autres utilisent l'anglais). Les différentes descriptions faites par les élèves à l'égard du français parlé à la maison sont résumées au tableau 1.

\section{Tableau 1}

\section{Description par les élèves du français parlé à la maison}

$\begin{array}{ll}\text { Nombre d'élèves } & \text { Description du français par les élèves } \\ 2 & \text { Français normal } \\ 2 & \text { Présence de mots anglais } \\ 3 & \text { Français plus standard } \\ 4 & \text { Chiac }\end{array}$

Quatre variétés de français semblent donc présentes dans les foyers et mènent à des représentations de valeurs différentes de la part des élèves. En réponse à la question portant sur la comparaison de leur langue parlée à la maison avec celle des autres familles de la région, certains élèves autoévaluent négativement leurs compétences en français, comme l'affirme cet élève : «Euh, ben je dirais que comme on est un ptit peu plus bas que zeux [les autres familles de la région qui parlent français] parce que comme on utilise des fois des langues, des mots anglais pour s'exprimer si on connaît pas le mot français... ». D'autres semblent plus positifs et sont d'avis qu'ils utilisent une variété de langue semblable à celle des autres familles de la région, bien que la comparaison à un français standard demeure à l'arrière-plan parmi les critères d'évaluation de leurs pratiques : « Euh, j'pense c'est comme la même chose dans les, comme les familles que je connais. J'pense pas qu'y n'a qui sont comme vraiment comme français français ». II est intéressant de noter (voir tableau 2) que 6 élèves sur les 14 interrogés ne répondent pas à cette question. Ce silence révèle-t-il une incapacité à effectuer une telle comparaison, ou s'agit-il d'une difficulté pour de jeunes adolescents à exprimer leur opinion sur le sujet dans un contexte qui leur est peu familier?

Après avoir comparé leurs propres pratiques langagières à celle des autres, les élèves ont été interrogés sur leurs sentiments par rapport à leur langue parlée. Le tableau 2 présente les valeurs positives ou négatives associées à ces comparaisons et à ces sentiments en relation avec le type de famille d'où proviennent les participants. 


\section{Tableau 2}

Valeur des comparaisons et des sentiments par rapport aux pratiques langagières en français à la maison, selon le type de mariage

\begin{tabular}{|l|l|l|l|}
\hline Nom & Type de mariage & Comparaisons & Sentiments \\
\hline Annie & Exogame & Sans réponse & Sans réponse \\
\hline Dominic & Exogame & Négatives & Négatifs \\
\hline Étienne & Exogame & Positives & Positifs \\
\hline Emmanuel & Endogame francophone & Sans réponse & Positifs \\
\hline Hélène & Endogame francophone & Positives & Positifs \\
\hline Gabriel & Exogame & Sans réponse & Positifs \\
\hline Josée & Endogame francophone & Sans réponse & Sans réponse \\
\hline Mathieu & Exogame & Sans réponse & Sans réponse \\
\hline Nicolas & Endogame francophone & Positives & Négatifs \\
\hline Richard & Exogame & Positives & Positifs \\
\hline Raphaël & Exogame & Sans réponse & Sans réponse \\
\hline Victoria & Endogame francophone & Mixtes & Mixtes \\
\hline Valérie & Endogame francophone & Négatives & Négatifs \\
\hline Virginie & Exogame & Positives & Sans réponse \\
\hline
\end{tabular}

Ainsi, chez les élèves interrogés, il ne semble pas exister de liens entre le type de famille, endogame ou exogame, et la valeur des comparaisons de leurs pratiques et des sentiments qu'ils expriment à l'égard du français parlé à la maison. Pour la plupart des élèves, les comparaisons et les sentiments sont de même valeur. II n'y a que le cas de Nicolas qui, même s'il se compare favorablement aux autres familles de la région, entretient tout de même des sentiments négatifs en ce qui a trait à sa langue. Les données recueillies dans cette étude ne permettent pas d'expliquer cette situation mais il semble que la valeur des comparaisons et des sentiments que les élèves expriment, ou non, est davantage reliée à leur vécu sociolangagier personnel plutôt qu'au type de famille dont ils sont issus.

Dans le contexte scolaire, 9 élèves affirment utiliser le français (les 5 autres disent continuer de parler chiac ou «les deux langues »). Certains élèves précisent qu'ils font un effort lorsqu'ils parlent aux enseignants : « ...je, j'essaie de plus utiliser un vocabulaire, euh, comme francophone avec mes enseignants... » et « J'parle plus français, pi des fois j'vas dire des mots anglais, comme si j'les connais pas en français ». D'autres élèves sont d'avis qu'il est important de faire un effort lorsqu'ils parlent aux enseignants parce que ces derniers exigent le français à l'école.

Dans le contexte de leurs cours de sciences, les élèves décrivent différemment le registre utilisé, comme le montre le tableau 3.

\section{Tableau 3}

\section{Description faites par les élèves du registre spécialisé utilisé en sciences}

$\begin{array}{ll}\text { Nombre d'élèves } & \text { Descriptions } \\ 3 & \text { Chiac } \\ 5 & \text { Vocabulaire particulier en sciences } \\ 6 & \text { Français plus « standard » }\end{array}$


Six élèves sont d'avis qu'un registre plus normatif doit être utilisé, alors que 3 autres élèves sont à l'aise d'utiliser le chiac. Cinq élèves sont conscients qu'il existe un vocabulaire spécifique au domaine scientifique, mais aucun élève ne semble être conscient de l'existence d'un registre spécialisé pour l'étude des sciences. D'ailleurs, certains élèves affirment qu'il n'existe aucun lien entre la langue et les sciences : « ...c'est pas vraiment un sujet about la langue... », « ...j'prends pas vraiment le cours de sciences comme un cours de français. »

\section{Représentations linguistiques du chiac}

Le chiac est une variété linguistique qui est utilisée par 12 élèves dans divers contextes. Les élèves décrivent cette variété de plusieurs façons, mais la plupart font allusion à la présence de mots anglais dans leur français. D'après les élèves, le chiac est une variété unique et facile à utiliser. Les élèves ne semblent pas se préoccuper au sujet de la norme avec le chiac. Chacun l'utilise comme bon lui semble. Les élèves affirment être à l'aise de le parler à la maison et avec leurs amis. Toutefois, selon certains, le chiac est difficilement compris à l'extérieur de la région. Les élèves semblent se représenter le chiac comme une variété qui peut être utilisée en famille et entre amis sans problème, mais qui ne peut pas être utilisée en tout temps. Par exemple, plusieurs élèves affirment que les francophones d'ailleurs ne les comprennent pas lorsqu'ils s'expriment en chiac.

\section{Représentations linguistiques de l'anglais}

Comme indiqué au tableau 4, l'anglais est une langue surtout utilisée par les élèves issus de mariages exogames. En fait, 10 élèves utilisent l'anglais à la maison ou avec leurs amis, dont 7 sont issus de mariages exogames. L'anglais est une langue qui semble surtout perçue comme étant nécessaire pour se débrouiller dans la vie de tous les jours.

\section{Tableau 4}

Présence de l'anglais dans les foyers et avec les amis, en fonction du type de mariage

\begin{tabular}{|l|l|l|l|c|}
\hline Nom & Type de mariage & $\begin{array}{l}\text { Première langue } \\
\text { apprise }\end{array}$ & $\begin{array}{l}\text { Parle anglais à } \\
\text { l'occasion à la } \\
\text { maison }\end{array}$ & $\begin{array}{l}\text { Parle anglais à } \\
\text { l'occasion avec ses } \\
\text { amis }\end{array}$ \\
\hline Annie & Exogame & Français & $\checkmark$ & $\checkmark$ \\
\hline Dominic & Exogame & Anglais & $\checkmark$ & \\
\hline Étienne & Exogame & Anglais & $\checkmark$ & $\checkmark$ \\
\hline Emmanuel & Endogame francophone & Français & & \\
\hline Hélène & Endogame francophone & Français & & $\checkmark$ \\
\hline Gabriel & Exogame & Français & $\checkmark$ & $\checkmark$ \\
\hline Josée & Endogame francophone & Fraçais & & $\checkmark$ \\
\hline Mathieu & Exogame & Français & $\checkmark$ & $\checkmark$ \\
\hline Nicolas & Endogame francophone & Français & & $\checkmark$ \\
\hline Richard & Exogame & Français & $\checkmark$ & \\
\hline Raphaël & Exogame & Anglais & $\checkmark$ & \\
\hline Victoria & Endogame francophone & Français & $\checkmark$ & \\
\hline Valérie & Endogame francophone & Français & & \\
\hline Virginie & Exogame & Français & & \\
\hline
\end{tabular}


En effet, certains élèves issus de mariages endogames francophones choisissent de parler anglais avec leurs amis : « Oui, parce que ça, ça comme, ça fait plus de sens parce que y [les amis] sont habitués à parler anglais à la maison pi ça, donc... ». Ces élèves croient que l'anglais est la langue avec laquelle leurs amis sont le plus à l'aise et les accommodent en conséquence. On remarque aussi, dans le tableau 4, que 8 élèves utilisent l'anglais à la maison, dont une élève issue d'un mariage endogame francophone. Cette élève précise que son père utilise l'anglais à la maison lorsqu'il travaille.

\section{Navigation entre les différentes langues et variété de langue selon le contexte}

Les élèves doivent souvent naviguer entre deux langues et la variété de langue qu'est le chiac dans un même contexte. Dans le contexte familial/social, ce phénomène est très fréquent. Les élèves utilisent le français, l'anglais et le chiac couramment et passent constamment de l'un à l'autre. Cependant, dans leurs propos, les élèves distinguent difficilement les frontières entre ces différentes langues et la variété "chiac». Par exemple, beaucoup d'élèves affirment utiliser le français, le chiac et l'anglais à la maison, et pour certains, la nuance entre le chiac et le français demeure vague. II est difficile de tracer une frontière bien définie, permettant de voir où s'arrête le français et où débute le chiac. Pour les élèves issus de mariages exogames, un mélange de français et d'anglais est utilisé à la maison de manière tellement naturelle qu'ils ne s'en rendent pas compte : « Euh, chepas, c'est normal à cause ça tout le temps été de même ", "...chepas, j'ai comme, j'ai pas de problèmes avec les deux [langues], comme j'peux switcher ${ }^{2}$ de un à l'autre ». II semble donc que lorsque l'on utilise différentes langues à l'intérieur même d'un foyer, il devient plus difficile pour les élèves de les isoler et de les différencier.

Dans le contexte familial/social, la navigation entre les langues et la variété « chiac » se fait de manière très naturelle. Les élèves passent de l'une à l'autre sans parfois même s'en rendre compte. Dans les autres contextes, toutefois, on remarque quelques inconforts quant à l'usage d'un français soutenu ou scientifique à l'école. Certains élèves semblent bien différencier entre les langues, la variété chiac et les registres propres à certains contextes et affirment les utiliser dans les situations appropriées, mais parfois avec quelques difficultés. Pour d'autres cependant, les frontières sont très floues. On se demande si ces élèves ne sont tout simplement pas conscients de l'existence de différents registres ou, s'ils en sont conscients, s'ils choisissent de ne pas les utiliser?

\section{Vécu des élèves lors des activités intégrant la langue en sciences}

La construction de récits narratifs a permis d'explorer le vécu de 3 élèves afin de tenter de décrire leurs comportements langagiers lors des activités intégrant la langue en sciences. Ces élèves ont un vécu sociolangagier différent. Richard ${ }^{3}$ est issu d'un couple exogame et la langue dominante chez-lui est l'anglais. Les parents de Nicolas sont tous les deux francophones et viennent de l'extérieur de l'Acadie. Emmanuel vit dans une famille endogame acadienne qui parle surtout le chiac. Les tableaux 2 et 4 fournissent également des informations au sujet de leurs représentations et pratiques langagières. En effet, Richard et Nicolas comparent leurs pratiques langagières positivement à celle des autres et Nicolas est le seul à entretenir des sentiments négatifs envers sa propre langue. Emmanuel parle surtout français au foyer et avec ses amis, alors que pour Richard, c'est l'anglais qui est la langue de

${ }^{2}$ Verbe anglais, conjugué en français de façon typiquement chiac, signifiant « changer »

${ }^{3}$ Les noms des élèves sont fictifs. 
choix. Nicolas parle surtout français à la maison, mais opte pour l'anglais avec ses amis. Ces élèves diffèrent également en termes de rendement scolaire. En nous basant sur nos observations et nos discussions avec les enseignants, Richard est un élève plus faible, Nicolas, un élève moyen, et Emmanuel, un élève plus fort.

En comparant le vécu de ces trois élèves, il est possible de relever certaines similarités. Premièrement, du côté des journaux réflexifs, les extraits des trois élèves se ressemblent beaucoup. Ils sont tous relativement courts et contiennent des informations portant essentiellement sur des faits appris pendant le cours. Si l'on interprète ces données en tenant compte des attentes normales associées aux niveaux de rendement de ces 3 élèves, personne ne ressort vraiment comme ayant utilisé cet outil pour réfléchir sur ses apprentissages. Ensuite, les trois élèves mentionnent, lors de l'entrevue, l'importance d'activités comme celles qu'ils ont vécues pour leur apprentissage. Toutefois, aucun élève ne relève les composantes langagières et leurs bienfaits. Ils affirment tout simplement que le travail de groupe et l'application des apprentissages ont été bénéfiques lors de telles activités.

En ce qui a trait à la discussion, l'observation montre qu'elle était beaucoup plus complexe du côté d'Emmanuel (l'élève plus fort) et de son groupe. Les élèves de ce groupe, incluant Emmanuel, proposaient des hypothèses et tentaient de les défendre. Ils apportaient des arguments et faisaient part de leur opinion. Dans les groupes où se trouvaient Richard et Nicolas, la discussion s'est avérée plutôt superficielle et même que, dans le groupe de Richard, l'aide-enseignante devait souvent mener la discussion et poser des questions afin de faire réfléchir les élèves. Ces résultats peuvent s'expliquer par le fait que les activités parmi lesquelles les élèves pouvaient choisir variaient selon le degré de difficulté; il est probable que des sous-groupes homogènes en termes de rendement scolaire se sont formés en fonction de la difficulté perçue des activités proposées, augmentant ainsi le potentiel de stimulation langagière pour Emmanuel et diminuant d'autant ce potentiel pour Nicolas et Richard.

Ainsi, les 3 élèves ont vécu des activités intégrant la langue en sciences, mais l'intégration de la langue a semblé passer inaperçue pour eux. Du moins, ils n'ont pas commenté sur l'apport des activités langagières sur leur apprentissage. Ils ne semblent pas avoir utilisé la langue comme outil d'apprentissage, pour clarifier leurs conceptions, décrire leur compréhension et confronter leurs idées. Sont-ils conscients que les stratégies langagières pourraient venir appuyer leurs apprentissages en sciences? Sont-ils plutôt surtout habitués à une pédagogie traditionnelle qui ne fait pas suffisamment de place aux activités réflexives langagières? Peut-être qu'une intégration d'activités langagières durant seulement 4 leçons n'était pas suffisante? Leurs représentations linguistiques à l'égard des registres soutenu en français (c'est une langue difficile) et spécialisé (les sciences, ce n'est pas un sujet à propos du français) entraventelles leur usage de la langue en sciences? En somme, l'analyse comparative des récits narratifs de ces trois élèves ne permet pas de conclure sur la relation entre leurs représentations linguistiques et leurs comportements langagiers en salle de classe, à cause probablement du manque de familiarité des élèves face aux activités littératiées en classe de sciences.

\section{Conclusion}

II importe, en milieu minoritaire, de comprendre comment les élèves se représentent les différents registres de langue auxquels ils sont exposés, afin d'assurer le succès des activités langagières en sciences. Puisque de telles activités sont essentielles à l'apprentissage des élèves en sciences, elles doivent être incluses dans l'enseignement 
quotidien. Toutefois, les représentations que les élèves se créent à l'égard des registres de langue qu'ils utilisent pourraient être un obstacle au bon fonctionnement des activités littératiées en sciences. En effet, les représentations linguistiques peuvent expliquer, en partie, le comportement langagier des élèves. Ainsi, un élève ayant une représentation négative de sa langue pourrait choisir de ne pas participer activement à une activité dans laquelle il doit s'exprimer oralement devant ses pairs.

Cette recherche visait à mieux comprendre comment des élèves du secondaire d'une région francophone minoritaire au Nouveau-Brunswick se représentaient divers registres de langue et comment ils vivaient des activités intégrant la langue en sciences. II ressort que les élèves utilisent couramment deux langues (le français et l'anglais) et une variété de langue (le chiac) dans leurs activités quotidiennes. Certains identifient clairement la langue ou la variété qu'ils utilisent dans un contexte particulier et peuvent choisir le registre approprié pour s'exprimer, alors que pour d'autres, les frontières sont très floues entre les différentes langues ou la variété chiac et ils ne semblent pas être conscients des différents registres langagiers. Chaque élève a son propre vécu sociolangagier et, en conséquence, se représente sa langue à sa manière. Certains autoévaluent leurs compétences langagières de manière positive et se sentent bien lorsqu'ils s'expriment, alors que pour d'autres, une insécurité linguistique semble être présente et pourrait leur causer certaines difficultés. Quant aux activités intégrant la langue en sciences, les élèves ne semblent pas avoir développé les compétences nécessaires pour les différencier des autres activités qu'ils vivent quotidiennement en classe. N'ayant pas l'habitude de vivre des activités littératiées en sciences, les élèves semblent incapables d'en reconnaître les bienfaits et d'en évaluer l'importance pour leur apprentissage. Ces résultats indiquent que des efforts accrus de conscientisation des enseignants restent à faire autant en ce qui a trait aux représentations linguistiques elles-mêmes, qu'en ce qui a trait à l'intégration d'activités littératiées en sciences.

Du côté des représentations linguistiques, les enseignants doivent reconnaître que les élèves utilisent plusieurs langues et la variété de langue chiac dans leurs divers contextes de vie. Yore (2008) affirme, dans sa théorie sur les registres de langue, que les élèves ont tous un registre vernaculaire, $L 1$, et qu'ils doivent naviguer entre ce registre et les autres registres utilisés quotidiennement comme le registre soutenu favorisé par l'école (L2) et le registre spécialisé des sciences (L3). Rivard et Cormier (2008) ajoutent qu'en milieu minoritaire, les élèves sont également exposés à la langue de la majorité, et que dans une même situation, l'élève navigue entre sa langue minoritaire (I1) et une langue majoritaire (L1). Toutefois, les résultats de notre recherche montrent que les élèves du sud-est du Nouveau Brunswick naviguent en plus entre le français et le chiac. Les diverses représentations du chiac dans le registre vernaculaire et leur interaction avec les représentations de la langue dans les registres scolaire et scientifique demande à être davantage explorées et prises en compte à l'école.

En sciences, on sait que l'acquisition du langage scientifique est essentielle à l'apprentissage et qu'une difficulté à ce niveau pourrait entraîner une difficulté au plan du raisonnement (Wellington et Osborne, 2001). Les élèves étudiés ici ne semblent toutefois pas reconnaître spontanément l'existence et l'importance du registre de langue spécialisé utilisé en sciences. Par conséquent, il revient aux enseignants d'intervenir directement pour orienter la construction de représentations positives de ce registre langagier par les élèves. Dans cette optique, les enseignants pourraient entretenir des discussions ouvertes avec les élèves concernant leurs représentations linguistiques en 
général, et l'apport plus spécifique des stratégies langagières pour enrichir leur apprentissage en sciences. II va sans dire que ces discussions devraient être complémentées de la planification d'activités littératiées régulières intégrées aux cours de sciences.

Nous reconnaissons que les adolescents ont sans doute de la difficulté à décrire leurs représentations linguistiques. II semble difficile pour eux d'être conscients de leurs représentations à l'égard des registres de langue qu'ils utilisent et de les partager. Nous reconnaissons aussi que la courte durée des activités intégrant la langue en sciences et le manque d'enseignement explicite de stratégies langagières (comment profiter d'un journal réflexif, comment trouver l'idée importante d'un texte, comment débattre une idée...) ont limité l'exploration du lien entre le succès ou le non succès de ces activités et les représentations linguistiques. Ainsi, de futures recherches sont de mise pour continuer l'exploration des représentations linguistiques dans les matières scolaires et surtout, pour élucider la question de la relation entre les représentations linguistiques et la qualité de la participation aux activités littératiées en sciences.

\section{Références}

Blanchet, P. et Robillard, D. (2003). Langues, contacts, complexité. Perspectives théoriques en sociolinguistique. [Cahiers de sociolinguistique $n$ 8]. Rennes : Presses universitaires de Rennes.

Boudreau, A. (1996). Les mots des jeunes Acadiens et Acadiennes du Nouveau-Brunswick. Dans L. Dubois et A. Boudreau (dir.), Les Acadiens et leur(s) langue(s) : quand le français est minoritaire (p. 137-155). Moncton : Les éditions d'Acadie.

Boudreau, A. et Perrot, M.-E. (2005). Quel français enseigner en milieu minoritaire? Minorités et contact de langues: le cas de I'Acadie. Glottopol, Revue de sociolinguistique en ligne, 6, 7-21.

Boudreau, A., Dubois, L. et d'Entremont, V. (2008). Représentations, sécurité/insécurité et éducation en milieu minoritaire, Dans P. Dalley et S. Roy (dir.), Francophonie, minorités et pédagogie (p. 45-175). Ottawa : Presses de l'Université d'Ottawa.

Bourdieu, P. (1982). Ce que parler veut dire. L'économie des échanges linguistiques. Paris : Fayard.

Buissière, P., Knighton, T. et Pennock, D. (2007). À la hauteur : Résultats canadiens de l'étude PISA de l'OCDE. Ottawa : Ministère de l'Industrie. [En ligne]. Disponible le 25 août, 2010 : http://www.pisa.gc.ca/81-590-F.pdf

Calvet, J.L. (1999). Pour une écologie des langues du monde. Paris : Éditions Plon.

Conseil des Ministres de l'éducation du Canada. (2004a). Rapport sur l'évaluation en sciences III; Programme d'indicateurs du rendement scolaire. [En ligne]. Disponible le 25 août, 2010 : http://www.cmec.ca/Programs/assessment/pancan/saip2004/Documents/SAIP-Science2004.fr.pdf

Cormier, M. (2004). Intégration des sciences et de la langue : création et expérimentation d'un modèle pédagogique pour améliorer l'apprentissage des sciences en milieu francophone minoritaire. Thèse de doctorat inédite, Université de Moncton, Moncton.

Cormier, M. (2005). La pédagogie en milieu minoritaire francophone : une recension des écrits. Moncton : Institut canadien de recherche sur les minorités linguistiques.

Cormier, M., Pruneau, D. et Rivard, L. (2004). S'approprier un vocabulaire scientifique en milieu minoritaire. Cahiers francocanadiens de l'ouest. 16 (1 et 2), 175-197.

Daley, P. (2003). Le rôle de reproduction sociale de l'enseignante et de l'école : deux paradoxes en classe de première année en Acadie du Nouveau-Brunswick. Dans H. Duchesne (dir.), Recherches en éducation francophone en milieu minoritaire: regards croisés sur une réalité mouvante (p. 1-22). Winnipeg: Presses universitaires de Saint-Boniface.

Deslauriers, J-P. (1991). Recherche qualitative : guide pratique. Montréal : Éditions McGraw-Hill.

Francart, M. (1997). Insécurité linguistique. Dans M.-L. Moreau (dir.), Sociolinguistique: Les concepts de base (p. 170-176). Paris : Pierre Mardaga.

Gee, J. (2002). Decontextualized language, a problem, not a solution. Paper presented at the Symposium : Ontological, Epistemological, Linguistic and Pedagogical Considerations of Language and Science Literacy : Empowering Research and Informing Instruction, September 15-20, 2002, Victoria, B. C.

Gee, J. (2008). Social linguistics and literacies: ideology in discourses. New York : Routledge. 
Gibbons, P. (1998). Classroom Talk and the Learning of New Registers in a Second Language. Language and Education, 12 (2), 99-118.

Hamers, J.F. et Blanc, M.H.A. (2000). Bilinguality and bilingualism. Cambridge : Cambridge University Press.

Hammond, W.J. (2002). The creative Journal : A power tool for learning. Green Teacher, 69, 34-38.

Hand, B. et Prain, V. (2006). Teachers Implementing Writing-to-Learn Strategies in Junior Secondary Science : A Case Study. Science Education, 90, 737-755.

Karsenti, T et Savoie-Zajc, L. (2004). La recherche en éducation : étapes et approches. Québec : Éditions du CRP.

Klinkenberg, J.-M. (2001). La langue et le citoyen. Paris : PUF.

Labov, W. (1972). Sociolinguistic patterns. Philadelphie : University of Pennsylvania Press.

Landry, R. et Allard, R. (1999). L'éducation dans la francophonie minoritaire. Dans J. Y. Thériault (dir.), Francophonies minoritaires au Canada : l'état des lieux (p. 403-434). Moncton : Les Éditions d'Acadie.

Landry, R. (2003a). Libérer le potentiel caché de l'exogamie : Profil démolinguistique des enfants des ayants droit francophones selon la structure familiale (Là où le nombre le justifie...IV). Moncton : Institut canadien de recherche sur les minorités linguistiques.

Landry, R. (2003b). Pour une pédagogie actualisante et communautarisante en milieu minoritaire francophone. Dans R. Allard. (dir.), Actes du colloque pancanadien sur la recherche en éducation en milieu francophone minoritaire : Bilan et prospective (p. 135-156). Québec : Association canadienne d'éducation de langue française.

Laplante, B. (2001). Des élèves de sixième année apprennent à parler des réactions chimiques. Dans D. Masny (Dir.), La culture de l'écrit : Les défis à l'école et au foyer (p. 105-141). Montréal, Québec : Éditions Logiques.

Laplante, B. (2007). Enseigner les sciences en montrant aux élèves à « parler sciences ». Dans P. Potvin, M. Riopel et S. Masson (dir.), Regards multiples sur l'enseignement des sciences (p. 254-265). Québec : Éditions Multimondes.

Lee, O. et Fradd, S. (1996). Literacy skills in science learning among linguistically diverse students. Science Education, 80 (6), 651-671.

Legendre, R. (2005). Dictionnaire actuel de l'éducation. Montréal : Guérin.

Mayan, M.J. (2001). An introduction to qualitative methods : A training module for students and professionals. Edmonton : International Institute for Qualitative Methodology.

Norris, S.P., et Phillips, L.M. (2003). How literacy in its fundamental sense is central to scientific literacy. Science Education, 87 (2), 224-240.

Osborne, J. (2002). Science without literacy : A ship without a sail? Cambridge Journal of Education, 32 (2), 203-218.

Paillé, P. et Mucchielli, A. (2008). L'analyse qualitative en sciences humaines et sociales. Paris : Armand Colin.

Perrot, M.E. (2006). Statut et fonction symbolique du chiac : analyse de discours épilinguistiques. Francophonies d'Amérique, 22, 140-151.

Poisson, Y. (1991). La recherche qualitative en éducation. Montréal : Presses de l'Université du Québec.

Pruneau, D. et Langis, J. (2003). L'enseignement et l'apprentissage des sciences en milieu minoritaire : défis et possibilités. Dans R. Allard (dir.), Actes du colloque pancanadien sur la recherche en éducation en milieu francophone minoritaire ( $p$. 209-215). Québec : Association canadienne d'éducation de langue française.

Rivard, L.P. et Straw, S.B. (2000). The effect of talk and writing on learning science : An exploratory study. Science Education, 84(5), 566-593.

Rivard, L.P. (1994). A Review of Writing to Learn in Science : Implications for Practice and Research. Journal of Research in Science Teaching, 31(9), 969-983.

Rivard, L.P. et Cormier, M. (2008). Teaching Science to French Speaking Students in English Canada Using an Instructional Congruence Model Involving Discourse-Enabling Strategies. L1 - Educations Studies in Language and Literature, 8 (1), 2341.

Rocque, J. (2008). La vitalité des écoles de langue française en milieu francophone minoritaire : droit fondé sur les luttes du passé. Le point en administration de l'éducation, 11(2), 36-39.

Wellington, J. et Osborne, J. (2001). Language and Literacy in Science Education. Philadelphia : Open University Press.

Yore, L., Hand, B. et Prain, V. (2002). Scientists as Writers. Science Education, 84, 672-692.

Yore, L.D. (2008). Science literacy for all students : Language, culture, and knowledge about nature and naturally occurring events. L1 - Educational Studies in Language and Literature, 8 (1), 5-21. 